\title{
Special Units in Real Cyclic Sextic Fields
}

\author{
By Marie-Nicole Gras
}

Dedicated to Daniel Shanks

\begin{abstract}
We study the real cyclic sextic fields generated by a root $w$ of $(X-1)^{6}-$ $\left(t^{2}+108\right)\left(X^{2}+X\right)^{2}, t \in \mathbf{Z}-\{0, \pm 6, \pm 26\}$. We show that, when $t^{2}+108$ is square-free (except for powers of 2 and 3 ), and $t \neq 0, \pm 10 . \pm 54$, then $w$ is a generator of the module of relative units. The details of the proofs are given in [3].
\end{abstract}

1. Introduction. The "simplest cubic fields" of D. Shanks [7] are generated by a root $w_{3}$ of

$$
X^{3}-t X^{2}-(t+3) X-1, \quad t \in \mathbf{Z}
$$

and a conjugate of $w_{3}$ is $\left(-w_{3}-1\right) / w_{3}$.

We have given in [4] an analog for cyclic quartic fields; these fields are generated by a root $w_{4}$ of

$$
X^{4}-t X^{3}-6 X^{2}+t X+1, \quad t \in \mathbf{Z}-\{0, \pm 3\},
$$

and a conjugate of $w_{4}$ is $\left(w_{4}-1\right) /\left(w_{4}+1\right)$.

The main interest of these fields is that they have an explicit system of fundamental units and a relatively large class number. These class numbers have been computed in [7] for the cubic, and in [4] for the quartic case. A simplest cubic field is used in [6] to compute the first example where the real part of the class number of the $p$ th cyclotomic field is larger than $p$; similarly, the corresponding quartic fields are used in [1] to prove a general inequality for the real part of the class number of some cyclotomic fields.

Real cyclic sextic fields are studied in [5], where a table of units and class numbers (for conductors less than 2021) is computed. Here we define sextic fields in which we can find $\theta$ such that a conjugate of $\theta$ is a homographic function of $\theta$ (in fact, this conjugate is $(\theta-1) /(\theta+2))$, but this element, even if it is a unit, is not a relative unit, as it was in cubic and quartic cases. From this element $\theta$, we construct a relative unit $w$ which is the analog of (1) and (2). First we recall some general results concerning relative units in real cyclic sextic fields, and then apply these results to our special family of units.

Received February 27, 1986.

1980 Mathematics Subject Classification. Primary 12A45, 12A35. 
2. Relative Units. Let $K / \mathbf{Q}$ be a real cyclic sextic field of conductor $f$, let $\sigma$ be a generator of its Galois group, and let $k_{2}$ and $k_{3}$ be the quartic and cubic subfields of $K$.

Let $E$ be the group of units of $K$. We consider the group of relative units of $K$ (i.e., the elements of $E$ whose norm is \pm 1 over $k_{2}$ and $k_{3}$ ); this group is $\langle \pm 1\rangle \oplus E_{*}$, where

$$
E_{*}=\left\{u \in E, u^{1-\sigma+\sigma^{2}}=1\right\} .
$$

Then $E_{*}$ is a free module over $\mathbf{Z}[\sigma] /\left(1-\sigma+\sigma^{2}\right) \simeq \mathbf{Z}[\exp (2 i \pi / 6)]$; this implies that there exists a generating relative unit, i.e., a unit $\varepsilon$ such that every unit $u \in E_{*}$ may be written

$$
u=\varepsilon^{\lambda+\mu \sigma}, \quad \lambda, \mu \in \mathbf{Z}
$$

moreover, if $\langle u\rangle$ is the submodule of $E_{*}$ generated by $u, u \neq 1$, then we have

$$
\left(E_{*}:\langle u\rangle\right)=\lambda^{2}+\lambda \mu+\mu^{2} .
$$

Using the general formula established in [2], we obtain an upper bound for $\left(E_{*}:\langle u\rangle\right)$ which is of the same kind as the one of [5]:

THEOREM 1. If the conductor $f$ of $K$ is $\geqslant 28$, then for any unit $u \in E_{*}-\{1\}$ we have

$$
\left(E_{*}:\langle u\rangle\right) \leqslant M_{*}=\frac{16}{3} \frac{R_{*}(u)}{(\log ((f-20) / 4))^{2}},
$$

where

$$
R_{*}(u)=(\log |u|)^{2}+\left(\log \left|u^{\sigma}\right|\right)^{2}-(\log |u|)\left(\log \left|u^{\sigma}\right|\right) .
$$

3. A Family of Real Sextic Fields. Let $t \in \mathbf{Z}$; we consider the fields $K_{t}=\mathbf{Q}(\theta)$, where $\theta$ is a root of

$$
P=X^{6}-\frac{t-6}{2} X^{5}-5 \frac{t+6}{4} X^{4}-20 X^{3}+5 \frac{t-6}{4} X^{2}+\frac{t+6}{2} X+1 .
$$

We show in [3] the following properties $\left(\mathrm{P}_{1}\right)$ to $\left(\mathrm{P}_{5}\right)$ :

$\left(\mathrm{P}_{1}\right)$ If $t \in \mathbf{Z}-\{0, \pm 6, \pm 26\}$, then $P$ is irreducible in $\mathbf{Q}[X]$, and $K_{t}=\mathbf{Q}(\theta)$ is a real cyclic sextic field; a generator $\sigma$ of its Galois group is characterized by the relation $\sigma(\theta)=(\theta-1) /(\theta+2)$. We have $K_{-t}=K_{t}$ for all $t \in \mathbf{Z}$, and then we can suppose that $t \in \mathbf{N}-\{0,6,26\}$.

$\left(\mathrm{P}_{2}\right)$ The quadratic subfield of $K_{t}$ is $k_{2}=\mathbf{Q}\left(\sqrt{t^{2}+108}\right)$.

$\left(\mathrm{P}_{3}\right)$ The cubic subfield of $K_{t}$ is $k_{3}=\mathbf{Q}(\varphi)$, where

$$
\varphi=\theta^{-1-\sigma^{3}}=-\frac{2 \theta+1}{\theta(\theta+2)}
$$

and

$$
\operatorname{Irr}(\varphi, \mathbf{Q})=X^{3}-\frac{t-6}{4} X^{2}-\frac{t+6}{4} X-1 ;
$$

the discriminant of this polynomial is $\left(\left(t^{2}+108\right) / 16\right)^{2}$. 
$\left(\mathrm{P}_{4}\right)$ The conductor $f$ of $K$, is given by the following procedure: Let $m$ be the product of primes, different from 2 and 3, dividing $t^{2}+108$ with an exponent not congruent to 0 modulo 6 ; then $f=4^{k} 3^{l} m$, where

$$
\begin{aligned}
k=0 & \text { if } t \equiv 1 \bmod 2 \text { or } t \equiv \pm 6 \bmod 16, \quad k=1 \quad \text { if not, } \\
l=0 & \text { if } t \equiv 1 \bmod 3, \quad l=1 \quad \text { if } t \equiv 0 \bmod 27, \quad l=2 \quad \text { if not. }
\end{aligned}
$$

$\left(\mathrm{P}_{5}\right)$ If $t \equiv 2 \bmod 4$, the polynomial $P$ defined in (8) belongs to $\mathbf{Z}[X], \theta$ is a unit of $K_{t}$, and $k_{3}=\mathbf{Q}(\varphi)$, where

$$
\operatorname{Irr}(\varphi, \mathbf{Q})=X^{3}-n X^{2}-(n+3) X-1, \text { with } n=(t-6) / 4 ;
$$

then the fields $k_{3}$ are the "simplest cubic fields" recalled in (1).

4. The Group $E_{*}$ for the Fields $K_{t}$. We consider the following element of $K_{t}$ :

$$
w=\theta^{1-\sigma^{3}}=-\frac{\theta(2 \theta+1)}{\theta+2} ;
$$

then $w^{\sigma}=-\theta(\theta-1) /(\theta+1)(\theta+2)$, and we verify that $(t \in \mathbf{N}-\{0,6,26\})$

$$
\operatorname{Irr}(w, \mathbf{Q})=(X-1)^{6}-\left(t^{2}+108\right)\left(X^{2}+X\right)^{2}
$$

and, by construction, $w \in E_{*}$ (see (3)).

First, we study the size of $w$ when $t \rightarrow+\infty$; we show that $\theta \sim t / 2$, and then $w \sim-t$ and $w^{\sigma} \sim-1$. We deduce from this, that when $t \rightarrow+\infty$, we have

$$
R_{*}(w) \sim(\log t)^{2} .
$$

Next, we study if $w$ is a generator of $E_{*}$. By computing an upper bound for the roots of (10), and using (6), we obtain: If $t \geqslant 26$ and $f \geqslant 28$, then

$$
\left(E_{*}:\langle w\rangle\right) \leqslant M_{t}=\frac{16}{3}\left(\frac{\log (t+6)}{\log ((f-20) / 4)}\right)^{2} .
$$

Finally, using (4) and (5), we test if there exist $\lambda, \mu \in \mathbf{Z}$ such that $w=\varepsilon^{\lambda+\mu \sigma}$, for all $\lambda, \mu$ such that $\rho=\lambda^{2}+\lambda \mu+\mu^{2} \leqslant M_{t}$. The values of $\rho$ which are to be considered are then $\rho=3, \rho=4$ or $\rho \geqslant 7(\rho=7,9,13, \ldots)$ :

(i) We have $\rho=3$ if and only if there exists $v \in E_{*}$ such that $w=v^{1+\sigma}$, and we verify that this happens if and only if there exists $s \in \mathbf{Z}$ such that

$$
t=s\left(s^{2}+9\right) \text {. }
$$

(ii) We have $\rho=4$ if and only if there exists $u \in E_{*}$ such that $w=u^{2}$, and this is impossible, since $w$ is not totally positive; then the index $\rho$ is odd.

To apply (12), it is necessary to know the conductor $f$ of $K_{t}$ and then, using $\left(\mathrm{P}_{4}\right)$, the squares dividing $t^{2}+108$. So we obtain two infinite sets of fields $K_{t}$ where a generator of $E_{*}$ is known:

Definition 1. Let $T$ be the infinite set of $t \in \mathbf{N}, t \neq 0$, such that $t^{2}+108$ is square-free, except for powers of 2 and 3.

For all $t \in T$, we consider the field $K_{t}=\mathbf{Q}(w)$, where $w$ is defined in (10). The main result concerning this family is the following

THEOREM 2. For all $t \in T-\{10,54\}$, the unit $w$ given by $(10)$ is a generator of the module $E_{*}$ of relative units of $K_{t}$. 
Proof. We verify, using (13), that $\left(E_{*}:\langle w\rangle\right)=3$ if and only if $t=10(s=1)$ and $t=54(s=3)$. After this, we can prove, using (12), that for all $t>90,\left(E_{*}:\langle w\rangle\right)<7$, and we compute directly $\left(E_{*}:\langle w\rangle\right)$ for all fields $K_{t}, t \in T, t \leqslant 90$ [3].

Definition 2. Let $S$ be the infinite set of $s \in \mathbf{N}, s \neq 0,2$, such that $s^{2}+3$ and $s^{2}+12$ are square-free, except for powers of 2 .

For all $s \in S$, let $t=s\left(s^{2}+9\right)$; we consider the field $L_{s}=K_{t}=\mathbf{Q}(w)$, where $w$ is defined in (10). From (13), there exists a unit $v \in E_{*}$ such that $w=v^{1+\sigma}$, and we compute

$$
\operatorname{Irr}(v, \mathbf{Q})=(X-1)^{6}+\left(s^{2}+12\right)\left(X^{5}-X^{4}-s^{2} X^{3}-X^{2}+X\right) .
$$

We have $t^{2}+108=\left(s^{2}+3\right)^{2}\left(s^{2}+12\right)$; since $s \in S$, we deduce from $\left(\mathrm{P}_{4}\right)$ that the conductor $f$ of $L_{s}$ is

$$
f=\frac{\left(s^{2}+3\right)\left(s^{2}+12\right)}{e}, \text { where } e \text { divides } 48 .
$$

Let $M_{*}$ be the upper bound of $\left(E_{*}:\langle v\rangle\right)$ given in (6). When $s \rightarrow+\infty$, from (11) there follows $R_{*}(v) \sim \frac{1}{3}\left(\log s^{3}\right)^{2}$, and if $s \in S$, from (15) there follows $\log ((f-20) / 4) \sim \log s^{4}$. Then,

$$
M_{*} \rightarrow 1 \text { when } s \rightarrow+\infty \text { and } s \in S \text {. }
$$

This proves that the upper bound established in Theorem 1 cannot be improved for the set of real cyclic sextic fields.

In the same way, we prove in [3] the analog of Theorem 2 for the set $S$ :

THEOREM 3. For all $s \in S$, the unit $v$ given by (14) is a generator of the module $E_{*}$ of relative units of $L_{s}$.

Remark. If $s \in S$ and if $s=6 r+3$, then the conductor of $k_{2}$ is $f_{2}=36 r^{2}+36 r$ +21 , and the fundamental unit of $k_{2}$ is

$$
\varepsilon_{2}=\left(\left(12 r^{2}+12 r+5\right)+(2 r+1) \sqrt{\left(36 r^{2}+36 r+21\right)}\right) / 2 .
$$

Since $t=(6 r+3)\left(36 r^{2}+36 r+18\right) \equiv 2 \bmod 4$, the field $k_{3}$ is a simplest cubic field. Then, if $s \in S$ and $s \equiv 3 \bmod 6$, the three fields $k_{2}, k_{3}$, and $K_{t}$ have an explicit system of fundamental units.

Université de Besançon et CNRS

Faculté des Sciences

Laboratoire de Mathématiques

U. A. n 040741

F-25030 Besançon Cédex, France

1. G. Cornell \& L. C. Washington, "Class numbers of cyclotomic fields," J. Number Theory, v. 21, 1985, pp. 260-274.

2. G. Gras \& M.-N. Gras, "Calcul du nombre de classes et des unités des extensions abéliennes réelles de Q," Bull. Sci. Math. (2), v. 101, 1977, pp. 97-129.

3. M.-N. Gras, "Familles d'unités dans les extensions cycliques réelles de degré 6 de Q," Publ. Math. Besançon, 1984, $85-1985 / 86$.

4. M.-N. Gras, "Table numérique du nombre de classes et des unités des extensions cycliques réelles de degré 4 de Q," Publ. Math. Besançon, 1977/78, fasc. 2, pp. 1-26 \& 1-53.

5. S. MäKı, The Determination of Units in Real Cyclic Sextic Fields, Lecture Notes in Math., vol. 797, Springer-Verlag, Berlin and New York, 1980.

6. E. Seah, L. C. Washington \& H. C. Williams, "The calculation of a large cubic class number with an application to real cyclotomic fields," Math. Comp., v. 41, 1983, pp. 303-305.

7. D. Shanks, “The simplest cubic fields,” Math. Comp., v. 28, 1974, pp. 1137-1152. 